\title{
NEW METHOD FOR WEIGHTED LOW-RANK APPROXIMATION OF COMPLEX-VALUED MATRICES AND ITS APPLICATION FOR THE DESIGN OF 2-D DIGITAL FILTERS
}

\author{
Wu-Sheng Lu and Andreas Antoniou \\ Dept. of Elec. and Comp. Engineering \\ University of Victoria \\ Victoria, BC, Canada V8W 3P6
}

\begin{abstract}
The design of two-dimensional (2-D) digital filters can be accomplished using the singular-value decomposition (SVD) method proposed by the authors in the past. The method in its present form treats all the elements of the sampled frequency-response matrix uniformly. Although the method works very well, in certain applications improved designs can be achieved by preconditioning the frequencyresponse matrix in order to emphasize important parts and deemphasize unimportant parts of the matrix. The preconditioning can be achieved through the use of an optimal weighted low-rank approximation (WLRA). Current methods for WLRA provide only local solutions. In this paper, we propose a method that can be used to perform WLRA which is globally optimal for complex-valued matrices. The usefulness of the proposed method will be demonstrated by applying it to the design of 2-D digital filters.
\end{abstract}

\section{INTRODUCTION}

The singular-value decomposition (SVD) [1]-[3] has found numerous applications in the past [4]-[13]. In a two dimensional (2-D) filter design context, the SVD is applied to a complex-valued matrix $\boldsymbol{F}$ obtained by sampling the desired frequency response, which results in several pairs of singular vectors. It has been shown that the design of a 2-D digital filter can be accomplished by designing a set of 1-D filters whose frequency responses approximate the singular vectors [8]-[13]. Although the method works very well, in certain applications, improved designs can be achieved by preconditioning the frequency-response matrix in order to emphasize important parts and deemphasize unimportant parts of the matrix. The preconditioning can be achieved through the use of an optimal weighted low-rank approximation (WLRA).

The WLRA was considered in [14] for real-valued matrices and in [15] for complex-valued matrices. However, the methods proposed in [14][15] only produce suboptimal solutions. In this paper, we propose a method that can be used to perform WLRA which is globally optimal for complexvalued matrices. In essence, our method is based on two facts: First, if the weighting matrix in the WLRA is a trivial matrix whose elements are all equal to unity, then the SVD provides a globally optimal solution for the WLRA problem; second, the globally optimal solution is a continuous function with respect to the elements of the weighting matrix. The usefulness of the proposed method is demonstrated by applying it to the design of 2-D digital filters.

\section{REVIEW OF SVD}

The SVD of a complex-valued matrix $\boldsymbol{F} \in C^{M \times N}$ can be expressed as

$$
\boldsymbol{F}=\boldsymbol{U} \boldsymbol{\Sigma} \boldsymbol{V}^{H}=\sum_{k=1}^{r} \sigma_{k} \boldsymbol{u}_{k} \boldsymbol{v}_{k}^{H}
$$

where $\boldsymbol{U}=\left[\begin{array}{llll}\boldsymbol{u}_{1} & \boldsymbol{u}_{2} & \cdots & \boldsymbol{u}_{M}\end{array}\right]$ and $\boldsymbol{V}=\left[\begin{array}{llll}\boldsymbol{v}_{1} & \boldsymbol{v}_{2} & \cdots & \boldsymbol{v}_{N}\end{array}\right]$ are unitary matrices, $\boldsymbol{\Sigma}=\operatorname{diag}\left\{\sigma_{1}, \sigma_{2}, \ldots, \sigma_{r}, 0 \ldots 0\right\}$ with singular values $\sigma_{1} \geq \sigma_{2} \geq \cdots \geq \sigma_{r}>0$, and $r$ is the rank of matrix $\boldsymbol{F}$. An important property of the SVD is that it offers an optimal low-rank approximation of $\boldsymbol{F}$ in both Enclidean and Frobenius norm sense for any rank not larger than $r$. That is, for any integer $K$ between 1 and $r$,

$$
\min _{\operatorname{rank}\left(\hat{\mathbf{F}}_{K}\right)=K}\left\|\boldsymbol{F}-\hat{\boldsymbol{F}}_{K}\right\|_{2, F}=\left\|\boldsymbol{F}-\boldsymbol{F}_{K}\right\|_{2, F}
$$

where the optimal rank- $K$ approximation $\boldsymbol{F}_{K}$ is given by

$$
\boldsymbol{F}_{K}=\sum_{k=1}^{K} \sigma_{k} \boldsymbol{u}_{k} \boldsymbol{v}_{k}^{H}
$$

By writing $\tilde{\boldsymbol{u}}_{k}=\sigma_{k}^{1 / 2} \boldsymbol{u}_{k}$ and $\tilde{\boldsymbol{v}}_{k}=\sigma_{k}^{1 / 2} \boldsymbol{v}_{k}$, the SVD in (1) and the optimal rank- $K$ approximation in (3) can be expressed as

$$
\boldsymbol{F}=\sum_{k=1}^{r} \tilde{\boldsymbol{u}}_{k} \tilde{\boldsymbol{v}}_{k}^{H}
$$




$$
\boldsymbol{F}_{K}=\sum_{k=1}^{K} \tilde{\boldsymbol{u}}_{k} \tilde{\boldsymbol{v}}_{k}^{H}
$$

respectively.

\section{WLRA OF A COMPLEX-VALUED MATRIX}

Given a complex-valued matrix $\boldsymbol{F} \in C^{M \times N}$ and a realvalued weighting matrix $\boldsymbol{W} \in R^{M \times N}$ such that $\operatorname{rank}(\boldsymbol{F})=$ $r$, we seek vectors $\boldsymbol{u}_{k} \in C^{M \times 1}, k=1, \ldots, K$ and $\boldsymbol{v}_{k} \in$ $C^{N \times 1}, k=1, \ldots, K$ that minimize the error function

$$
J(\boldsymbol{x}, \boldsymbol{W})=\left\|\boldsymbol{W} \circ\left(\boldsymbol{F}-\sum_{k=1}^{K} \boldsymbol{u}_{k} \boldsymbol{v}_{k}^{H}\right)\right\|_{F}
$$

where

$$
\boldsymbol{x}=\left[\begin{array}{c}
\boldsymbol{u} \\
\boldsymbol{v}
\end{array}\right], \boldsymbol{u}=\left[\begin{array}{c}
\boldsymbol{u}_{1} \\
\vdots \\
\boldsymbol{u}_{K}
\end{array}\right], \boldsymbol{v}=\left[\begin{array}{c}
\boldsymbol{v}_{1} \\
\vdots \\
\boldsymbol{v}_{K}
\end{array}\right]
$$

$\boldsymbol{W} \circ \boldsymbol{Y}$ denotes elementwise multiplication of matrices $\boldsymbol{W}$ and $\boldsymbol{Y}$, and $K$ is an integer between 1 and $r$.

The function $J(\boldsymbol{x}, \boldsymbol{W})$ in (6) is a 4th-order function of variable $\boldsymbol{x}$. As such, the application of a general nonlinear optimization algorithm usually yields a local minimizer and the performance of the local minimizer depends largely on the choice of the optimization algorithm and the initial point [14][15]. However, there is an exception: if $\boldsymbol{W}_{0}$ is an allone matrix, then a global minimizer of function $J\left(\boldsymbol{x}, \boldsymbol{W}_{0}\right)$ in (6) is provided by the SVD of $\boldsymbol{F}$ [2].

\section{A GLOBAL SOLUTION OF WLRA}

\subsection{The Solution Method}

From (6), it follows that function $J(\boldsymbol{x}, \boldsymbol{W})$ is a quadratic function of the elements of matrix $\boldsymbol{W}$. Consequently, for a fixed $\boldsymbol{F}$, the global minimizer of $J(\boldsymbol{x}, \boldsymbol{W})$ is a continuous function of $\boldsymbol{W}$. In other words, if $\boldsymbol{x}^{*}(\boldsymbol{W})$ is the global minimizer of $J(\boldsymbol{x}, \boldsymbol{W})$ for a given $\boldsymbol{W}$, then the difference $\left\|\boldsymbol{x}^{*}(\boldsymbol{W}+\Delta \boldsymbol{W})-\boldsymbol{x}^{*}(\boldsymbol{W})\right\|$ can be made arbitrarily small if $\|\Delta \boldsymbol{W}\|$ is sufficiently small. Given a weighting matrix $\boldsymbol{W}$, let $\left\{\boldsymbol{W}_{l}, l=0,1 . \ldots, L\right\}$ be a sequence of weighting matrices that satisfies three conditions: (i) $\boldsymbol{W}_{0}$ is the all-one matrix, (ii) $\left\|\boldsymbol{W}_{l}-\boldsymbol{W}_{l+1}\right\|$ is small for $l=1, \ldots, L-1$, and (iii) $\boldsymbol{W}_{L}=\boldsymbol{W}$. In other words, $\left\{\boldsymbol{W}_{l}, l=0,1, \ldots, L\right\}$ is a sequence of matrices that interpolates between $\boldsymbol{W}_{0}$ and $W$ with sufficiently high interpolation density. To obtain a global minimizer of $J\left(\boldsymbol{x}, \boldsymbol{W}_{0}\right)$, we use the SVD of $\boldsymbol{F}$ to obtain a global minimizer of $J(\boldsymbol{x}, \boldsymbol{W})$ and denote the minimizer by $\boldsymbol{x}^{*}\left(\boldsymbol{W}_{0}\right)$. Next, point $\boldsymbol{x}^{*}\left(\boldsymbol{W}_{0}\right)$ is used as the initial point to minimize function $J\left(\boldsymbol{x}, \boldsymbol{W}_{1}\right)$. Since $\boldsymbol{W}_{1}$ is close to $\boldsymbol{W}_{0}, \boldsymbol{x}^{*}\left(\boldsymbol{W}_{1}\right)$ is close to $\boldsymbol{x}^{*}\left(\boldsymbol{W}_{0}\right)$. Moreover, since the optimization starts with $\boldsymbol{x}^{*}\left(\boldsymbol{W}_{0}\right)$, it is expected that the algorithm will converge to $\boldsymbol{x}^{*}\left(\boldsymbol{W}_{1}\right)$ in a small number of iterations. This can then serve as the initial point for the minimization of $J\left(\boldsymbol{x}, \boldsymbol{W}_{2}\right)$. This process is continued until the minimization of $J\left(\boldsymbol{x}, \boldsymbol{W}_{L}\right)$ is achieved.

\subsection{Minimization of $J\left(x, W_{l}\right)$}

A key step in the implementation of the above solution method is to minimize function $J\left(\boldsymbol{x}, \boldsymbol{W}_{l}\right)$ for each weighting matrix $\boldsymbol{W}_{l}$. Since the initial point $\boldsymbol{x}^{*}\left(\boldsymbol{W}_{l-1}\right)$ is not far from the solution, it is quite appropriate to consider perturbed vectors $\boldsymbol{u}_{k}+\gamma_{k}$ and $\boldsymbol{v}_{k}+\boldsymbol{\delta}_{k}$ for $k=1, \ldots, K$. In order to ensure that the perturbed vectors are not widely different from their unperturbed counterparts, the constraints

$$
\left\|\gamma_{k}\right\|_{\infty} \leq b_{k} \text { and }\left\|\boldsymbol{\delta}_{k}\right\|_{\infty} \leq b_{k} \quad \text { for } 1 \leq k \leq K
$$

are imposed where $b_{k}$ are prescribed upper bounds, and $\|\cdot\|_{\infty}$ denotes the infinity norm which is equal to the maximum magnitude of the vector's components. Under these conditions, the optimization problem at hand becomes

$$
\begin{gathered}
\underset{\boldsymbol{\gamma}, \boldsymbol{\delta}}{\operatorname{minimize}}\left\|\boldsymbol{W} \circ\left[\boldsymbol{F}-\sum_{k=1}^{K}\left(\boldsymbol{u}_{k}+\boldsymbol{\gamma}_{k}\right)\left(\boldsymbol{v}_{k}+\boldsymbol{\delta}_{k}\right)^{H}\right]\right\|_{F}^{2} \\
\text { subject to: }\left\|\boldsymbol{\gamma}_{k}\right\|_{\infty} \leq b_{k} \text { and }\left\|\boldsymbol{\delta}_{k}\right\|_{\infty} \leq b_{k} \\
\text { for } k=1, \ldots, K
\end{gathered}
$$

Since the perturbation vectors $\gamma_{k}$ and $\delta_{k}$ are all small in magnitude, the second-order term $\boldsymbol{W} \circ \sum_{k=1}^{K} \boldsymbol{\gamma}_{k} \boldsymbol{\delta}_{k}^{H}$ can be neglected and the objective function in (8a) can be approximated as

$$
\hat{J}(\boldsymbol{z}, \boldsymbol{W})=\left\|\boldsymbol{D}-\sum_{k=1}^{K} \boldsymbol{W} \circ\left(\boldsymbol{\gamma}_{k} \boldsymbol{v}_{k}^{H}\right)-\sum_{k=1}^{K} \boldsymbol{W} \circ\left(\boldsymbol{u}_{k} \boldsymbol{\delta}_{k}^{H}\right)\right\|_{F}^{2}
$$

where

$$
\begin{aligned}
& \boldsymbol{z}=\left[\begin{array}{l}
\boldsymbol{\gamma} \\
\boldsymbol{\delta}
\end{array}\right], \boldsymbol{\gamma}=\left[\begin{array}{c}
\gamma_{1} \\
\vdots \\
\gamma_{K}
\end{array}\right], \boldsymbol{\delta}=\left[\begin{array}{c}
\boldsymbol{\delta}_{1} \\
\vdots \\
\boldsymbol{\delta}_{K}
\end{array}\right] \\
& \boldsymbol{D}=\boldsymbol{W} \circ\left(\boldsymbol{F}-\sum_{k=1}^{K} \boldsymbol{u}_{k} \boldsymbol{v}_{k}^{H}\right)
\end{aligned}
$$

It can be verified that $\hat{J}(\boldsymbol{z}, \boldsymbol{W})$ in (9) is a quadratic function of $\boldsymbol{z}$ with a positive definite Hessian matrix. Consequently, the optimization problem.

$$
\begin{gathered}
\text { minimize } \hat{J}(\boldsymbol{z}, \boldsymbol{W}) \\
\text { subject to: constraints in (8b) }
\end{gathered}
$$

is a quadratic programming $(\mathrm{QP})$ problem. Efficient algorithms for QP problems are available in the literature [16][17]. 


\subsection{A Special Case}

A special case of practical importance is the WLRA for realvalued matrices. If $\boldsymbol{F} \in R^{M \times N}$, then the quadratic function $\hat{J}(\boldsymbol{z}, \boldsymbol{W})$ in (9) can be expressed as

$\hat{J}(\boldsymbol{z}, \boldsymbol{W})=\boldsymbol{z}^{T} \boldsymbol{Q} \boldsymbol{z}-2 \boldsymbol{z}^{T} \boldsymbol{q}+\|\boldsymbol{D}\|_{F}^{2}$

where

$$
\begin{aligned}
& \boldsymbol{Q}=\left[\begin{array}{cc}
\boldsymbol{E} & \boldsymbol{Y} \\
\boldsymbol{Y}^{T} & \boldsymbol{G}
\end{array}\right], \boldsymbol{q}=\left[\begin{array}{c}
\boldsymbol{q}_{\gamma} \\
\boldsymbol{q}_{\sigma}
\end{array}\right] \\
& \boldsymbol{E}=\left\{\boldsymbol{E}^{(k, m)}, 1 \leq k \leq K, 1 \leq m \leq K\right\} \\
& \boldsymbol{E}^{(k, m)}=\operatorname{diag}\left\{e_{1}^{(k, m)}, \ldots, e_{M}^{(k, m)}\right\} \\
& e_{i}^{(k, m)}=\sum_{j=1}^{N} w_{i j}^{2} v_{j}^{(k)} v_{j}^{(m)} \\
& \boldsymbol{G}=\left\{\boldsymbol{G}^{(k, m)}, 1 \leq k \leq K, 1 \leq m \leq K\right\} \\
& \boldsymbol{G}^{(k, m)}=\operatorname{diag}\left\{g_{1}^{(k, m)}, \ldots, g_{N}^{(k, m)}\right\} \\
& g_{j}^{(k, m)}=\sum_{i=1}^{M} w_{i j}^{2} u_{i}^{(k)} u_{i}^{(m)} \\
& \boldsymbol{Y}=\left\{\boldsymbol{Y}^{(k, m)}, 1 \leq k \leq K, 1 \leq m \leq K\right\} \\
& y_{i j}^{(k, m)}=w_{i j}^{2} u_{i}^{(m)} v_{j}^{(k)} \text { for } 1 \leq i \leq M, 1 \leq j \leq N \\
& q_{\gamma}=\left[\begin{array}{c}
\boldsymbol{q}_{\gamma 1} \\
\vdots \\
\boldsymbol{q}_{\gamma K}
\end{array}\right], q_{\delta}=\left[\begin{array}{c}
\boldsymbol{q}_{\delta 1} \\
\vdots \\
\boldsymbol{q}_{\delta K}
\end{array}\right] \\
& \boldsymbol{q}_{\gamma k}=(\boldsymbol{W} \circ \boldsymbol{D}) \boldsymbol{v}_{k} \\
& \boldsymbol{q}_{\delta k}=(\boldsymbol{W} \circ \boldsymbol{D})^{T} \boldsymbol{u}_{k}
\end{aligned}
$$

In the above formulas, $w_{i j}$ denotes the $(i, j)$ th element of $\boldsymbol{W}$ and $u_{i}^{(k)}$ denotes the $i$ th element of $\boldsymbol{u}_{k}$.

The constraints in $(8 b)$ become

$$
\left[\begin{array}{l}
-\boldsymbol{b}_{\gamma} \\
-\boldsymbol{b}_{\delta}
\end{array}\right] \leq\left[\begin{array}{l}
\gamma \\
\boldsymbol{\delta}
\end{array}\right] \leq\left[\begin{array}{l}
\boldsymbol{b}_{\gamma} \\
\boldsymbol{b}_{\delta}
\end{array}\right]
$$

where

$$
\boldsymbol{b}_{\gamma}=\left[\begin{array}{c}
b_{1} \boldsymbol{e}_{\gamma} \\
\vdots \\
b_{K} \boldsymbol{e}_{\gamma}
\end{array}\right], \boldsymbol{b}_{\delta}=\left[\begin{array}{c}
b_{1} \boldsymbol{e}_{\delta} \\
\vdots \\
b_{K} \boldsymbol{e}_{\delta}
\end{array}\right]
$$

$\boldsymbol{e}_{\gamma}=\left[\begin{array}{lll}1 & \cdots & 1\end{array}\right]^{T} \in R^{M \times 1}$ and $\boldsymbol{e}_{\delta}=\left[\begin{array}{lll}1 & \cdots & 1\end{array}\right]^{T} \in R^{N \times 1}$. Therefore, the QP problem in (10) becomes

$$
\begin{array}{r}
\operatorname{minimize} \boldsymbol{z}^{T} \boldsymbol{Q} \boldsymbol{z}-2 \boldsymbol{z}^{T} \boldsymbol{q} \\
\text { subject to: }-\boldsymbol{b} \leq \boldsymbol{z} \leq \boldsymbol{b}
\end{array}
$$

where $\boldsymbol{b}=\left[\begin{array}{ll}\boldsymbol{b}_{\gamma}^{T} & \boldsymbol{b}_{\delta}^{T}\end{array}\right]^{T}$. The problem in (12) involves $(M+$ $N) K$ variables and $2(M+N) K$ linear constraints.

The solution obtained by solving the problem in (12) is used to update vectors $\boldsymbol{u}$ and $\boldsymbol{v}$ (see (6a)). The updated $\boldsymbol{u}$ and $\boldsymbol{v}$ are then used to update $Q$ and $\boldsymbol{q}$ using (11b)-(11m), and the updated $\boldsymbol{Q}$ and $\boldsymbol{q}$ are used in the problem in (12) to find a new perturbation vector $z$. The iteration continues until the magnitude of $z$ is less than a prescribed tolerance.

\section{APPLICATION FOR THE DESIGN OF 2-D DIGITAL FILTERS}

For the sake of simplicity, we consider the design of linearphase 2-D digital filters. In this case, matrix $\boldsymbol{F}$ consists of the desired amplitude response at a set of frequency grid points over the region $\left\{-\pi \leq \omega_{1} \leq \pi,-\pi \leq w_{2} \leq \pi\right\}$. A piecewise constant weighting matrix $\boldsymbol{W}$ with large values for the passbands and stopbands and small values for the transition bands can be used to emphasize important parts and deemphasize unimportant parts of matrix $\boldsymbol{F}$. Having determined matrices $\boldsymbol{F}$ and $\boldsymbol{W}$, the method described in Sec. 4 can be applied for a given number of sections, $K$, to obtain vectors $\boldsymbol{u}_{k}, 1 \leq k \leq K$ and $\boldsymbol{v}_{k}, 1 \leq k \leq K$ that minimize $J(\boldsymbol{x}, \boldsymbol{W})$ in (6a).

The vectors $\boldsymbol{u}_{k}$ and $\boldsymbol{v}_{k}$ obtained can be viewed as the desired amplitude responses of a pair of 1-D digital filters. On the basis of these amplitude responses, corresponding transfer functions $F_{k}\left(z_{1}\right)$ and $G_{k}\left(z_{2}\right)$ can be obtained by solving the approximation problems involved. Using these transfer functions, a 2-D transfer function can be obtained as

$$
H\left(z_{1}, z_{2}\right)=\sum_{k=1}^{K} F_{k}\left(z_{1}\right) G_{k}\left(z_{2}\right)
$$

whose amplitude response approximates $\sum_{k=1}^{K} \boldsymbol{u}_{k} \boldsymbol{v}_{k}^{T}$.

The use of WLRA in the SVD method offers considerable design flexibility, in practice, and enables the designer to achieve not only a better design but also a more economical one.

\section{A DESIGN EXAMPLE}

The proposed method was applied to design a circularly symmetric, linear phase, lowpass, FIR 2-D filter. The normalized passband and stopband edges of the filter were set to $\omega_{p}=0.25$ and $\omega_{a}=0.35$, respectively. Because the filter is quadrantally symmetric, matrix $\boldsymbol{F}$ was composed of the desired magnitude response in the frequency region $\left\{0 \leq \omega_{1} \leq \pi,-\pi \leq \omega_{2} \leq 0\right\}$ over $31 \times 31$ grid points. The weighting function was given by

$$
W\left(\omega_{1}, \omega_{2}\right)= \begin{cases}1 & \text { for } 0 \leq \sqrt{\omega_{1}^{2}+\omega_{2}^{2}} \leq 0.26 \\ 0.1 & \text { for } 0.26<\sqrt{\omega_{1}^{2}+\omega_{2}^{2}}<0.35 \\ 1 & \text { for } \sqrt{\omega_{1}^{2}+\omega_{2}^{2}} \geq 0.35\end{cases}
$$


The sequence of interpolating weighting matrices, $\left\{\boldsymbol{W}_{i}, i=\right.$ $0,1, \ldots, 9\}$, were specified by

$$
W_{i}\left(\omega_{1}, \omega_{2}\right)= \begin{cases}1 & \text { for } 0 \leq \sqrt{\omega_{1}^{2}+\omega_{2}^{2}} \leq 0.26 \\ 1-0.1 i & \text { for } 0.26<\sqrt{\omega_{1}^{2}+\omega_{2}^{2}}<0.35 \\ 1 & \text { for } \sqrt{\omega_{1}^{2}+\omega_{2}^{2}} \geq 0.35\end{cases}
$$

For each $\boldsymbol{W}_{i}$, the MATLAB routine called quadprog was used to solve the QP problem in (12) and the algorithm described in Sec. 4.3 converged in 5 iterations. In our design, four pairs of linear-phase FIR 1-D filters of order 40 were used to construct the 2-D digital filter. The maximum passband ripple and minimum stopband attenuation of the 2-D filter were 0.0783 and $24.11 \mathrm{~dB}$, respectively. The amplitude response of the filter is shown in Fig. 1. For comparison purposes, a 2-D filter was obtained using the same design specifications by applying the SVD method [10][11] without WLRA. The maximum passband ripple and minimum stopband attenuation of the filter were 0.0814 and $22.76 \mathrm{~dB}$, respectively, and the amplitude response was similar to that in Fig. 1. In effect, significant improvements have been achieved through the use of weighting.

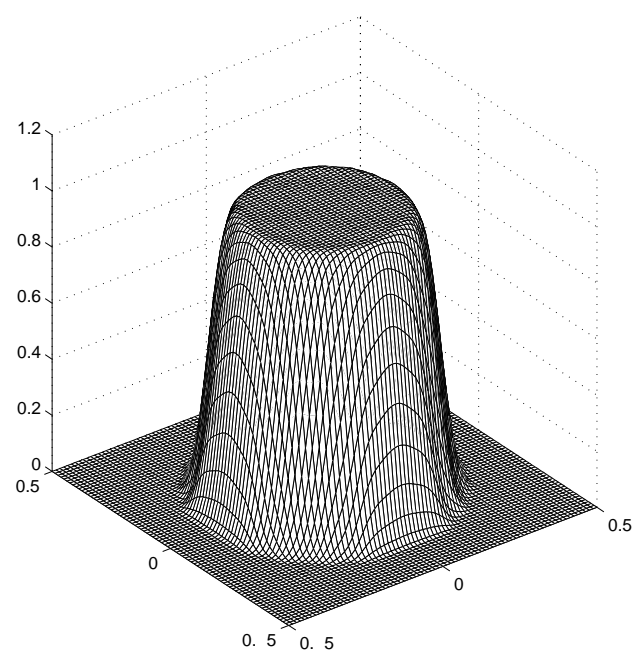

Figure 1: Amplitude response of the filter designed using the proposed method.

\section{CONCLUSIONS}

We have developed a method that can be used to perform WLRA that is globally optimal for complex-valued matrices. It has been shown that the global solution can be obtained by solving QP subproblems in an iterative manner. The usefulness of the proposed method has been demonstrated by showing that it yields designs with reduced passband ripple and increased stopband attenuation relative to the SVD method without WLRA.

\section{REFERENCES}

[1] R. A. Horn and C. R. Johnson, Matrix Analysis, Cambridge, UK: Cambridge University Press, 1985.

[2] G. W. Stewart, Introduction to Matrix Computations, New York, NY: Academic Press, 1974.

[3] G. H. Golub and C. F. Van Loan, Matrix Computations, 2nd edition, Baltimore, MD: Johns Hopkins University Press, 1989.

[4] V. C. Klema and A. J. Laub, "The singular value decomposition: Its computation and some applications", IEEE Trans. Automat. Contr., vol. 25, pp. 164-176, April 1980.

[5] T. Yoshikawa, Foundations of Robotics, Cambridge, MA: MIT Press, 1990.

[6] A. K. Jain, Fundamentals of Digital Image Processing, Englewood Cliffs, NJ: Prentice Hall, 1989.

[7] L. L. Scharf, "The SVD and reduced rank signal processing”, Signal Processing, vol. 25, pp. 113-133, Nov. 1991.

[8] S. Treitel and J. L. Shanks, "The design of multistage separable planar filters", IEEE Trans. Geosci. Electron, vol. 9, pp. 10-27, 1971.

[9] R. E. Twogood and S. K. Mitra, "Computer-aided design of separable two-dimensional digital filters", IEEE Trans. Acoust. Speech, Signal Processing, vol. 25, pp. 165-169, Feb. 1977.

[10] A. Antoniou and W.-S. Lu, "Design of 2-D digital filters by using the singular value decomposition", IEEE Trans. Circuits Syst., vol. 34, pp. 1191-1198, Oct. 1987.

[11] W. -S. Lu, H. -P. Wang, and A. Antoniou, "Design of 2D digital filters using the singular value decomposition and balanced approximation", IEEE Trans. Signal Processing, vol. 39, pp. 2253-2262, Oct. 1991.

[12] T. B. Deng and M. Kawamata, "Frequency domain design of 2-D digital filters using the iterative singular value decomposition", IEEE Trans. Circuits Syst., vol. 38, pp. 1225-1228, Oct. 1991.

[13] N. Yazdi, M. Ahmadi, and J. J. Soltis, "Efficient design of 2-D linear phase FIR filters using singular value decomposition", Elect. Lett., vol. 38, No. 24, pp. 2256-2258, Nov. 1992.

[14] D. Shpak, "A weighted-least-squares matrix decomposition method with application to the design of two-dimensional digital filters", in Proc. IEEE Midwest Symp. Circuits Syst., pp. 1070-1073, Calgary, Aug. 1990.

[15] W.-S. Lu, S.-C. Pei, and P.-H. Wang, "Weighted low-rank approximation of general complex matrices and its application in the design of 2-D digital filters," IEEE Trans. Circuits Syst., I, vol. 44, pp. 650-655, July 1997.

[16] D. G. Luenberger, Linear and Nonlinear Programming, 2nd edition, Reading, MA: Addison-Wesley, 1984.

[17] R. Fletcher, Practical Methods of Optimization, 2nd edition, Chichester, UK: John Wiley, 1987. 